Journal of Nonlinear Mathematical Physics

\title{
Exact Travelling Wave Solutions of a Beam Equation
}

J. C. Camacho, M. S. Bruzón, J. Ramírez, M. L. Gandarias

To cite this article: J. C. Camacho, M. S. Bruzón, J. Ramírez, M. L. Gandarias (2011) Exact Travelling Wave Solutions of a Beam Equation, Journal of Nonlinear Mathematical Physics 18:Supplement 1, 33-49, DOI: https://doi.org/10.1142/S140292511100126X

To link to this article: https://doi.org/10.1142/S140292511100126X

Published online: 04 January 2021 


\title{
EXACT TRAVELLING WAVE SOLUTIONS OF A BEAM EQUATION
}

\author{
J. C. CAMACHO, M. S. BRUZÓN, J. RAMÍREZ and M. L. GANDARIAS* \\ Departamento de Matemáticas, Universidad de Cádiz, P.O. Box 40 \\ Puerto Real, Cádiz 11510, Spain \\ *marialuz.gandarias@uca.es
}

Received 30 September 2010

Accepted 2 November 2010

\begin{abstract}
In this paper we make a full analysis of the symmetry reductions of a beam equation by using the classical Lie method of infinitesimals and the nonclassical method. We consider travelling wave reductions depending on the form of an arbitrary function. We have found several new classes of solutions that have not been considered before: solutions expressed in terms of Jacobi elliptic functions, Wadati solitons and compactons. Several classes of coherent structures are displayed by some of the solutions: kinks, solitons, two humps compactons.
\end{abstract}

Keywords: Beam equation; partial differential equation; symmetries.

Mathematics Subject Classification: 35D05; 70G65

\section{Introduction}

Historical events of travelling wave behavior in the Golden Gate Bridge in San Francisco motivated McKenna and Walter [25] to study travelling wave solutions in a nonlinear beam equation

$$
\Delta \equiv u_{t t}+u_{x x x x}+f(u)=0,
$$

where $u=u(x, t)$ is the deflection of the roadbed, the $x$-axis points are in the direction along the bridge and $t$ is time. The cables can apply some force even if they are loose and the force is given by some nonlinear function $f$, [10]. Suspension bridges have a history of large-scale oscillations caused by wind, earthquakes or traffic forces which may lead to structural failure. As a result of these oscillations and the resonance effects, the cables start to loosen and tighten producing a nonlinear effect.

Several papers investigated this beam equation from different points of view. In $[11,12]$ Chen and McKenna gave a variational proof, via the Mountain Pass Lemma, of the existence of travelling wave solutions for Eq. (1.1). They showed the existence of at least one nontrivial solution of (1.1). For $f(u)=e^{u-1}-1$, but by applying the Mountain Pass algorithm to a finite subinterval of $R$ they did obtain numerical solutions. They claimed that solutions seem to exist in the range $0<c<\sqrt{2}$. As the wave speed approaches $\sqrt{2}$, the solutions 
became highly oscillatory in nature, whereas when $c$ approaches 0 , they appear to go to infinity in amplitude.

In $[9,19,21]$ the authors studied Eq. (1.1) numerically and they used either continuation methods or variational numerical methods to gain more information on the structure of the equation solutions set. In $[20,24]$ the authors have considered the existence of multiple solutions for the beam equation. In $[14,15]$ the beam equation is transformed into an ordinary differential equation and is treated by using the considerable power of dynamical systems.

There is no existing general theory for solving nonlinear partial differential equations and the methods of point transformations are a powerful tool. Some of the most useful point transformations are those which form a continuous group. Lie classical symmetries admitted by nonlinear partial differential equations are useful for finding invariant solutions. Moreover symmetry reductions and exact solutions have several different important applications in the context of differential equations. Since solutions of partial differential equations asymptotically tend to solutions of lower-dimensional equations obtained by symmetry reductions, some of these special solutions will illustrate important physical phenomena. Motivated by the fact that symmetry reductions for many PDE's can not be obtained by using classical symmetries, there have been several generalizations of the classical Lie group method for symmetry reductions. The nonclassical method was firstly introduced by Bluman and Cole [4] to study the symmetry reductions of the heat equation. The description of the method can be found in $[4,5,17,22]$.

For several classes of PDE, the search for soliton-like solutions has raised a great interest during the last two decades. Solitons are analytical solutions that are exponentially localized in the space, but, in general, they are not null out of any bounded set. By weakening the regularity conditions and by strengthening the conditions of localization, the compactons were introduced $[28,29]$. The functions in this class are null out of some bounded spatial set but may not be analytical at some points.

For nonlinear PDE whose coefficients can vanish at some points, as is our beam model, the existence and relevance of non-analytical solutions has been considered by several authors. However, not always some of these authors use the same definitions to deal with compactons. The main differences appear with the behaviour of the possible solutions at the singularities of the equation. In this paper we understand that a compacton is a function that has enough continuous derivatives in order for this function to satisfy the PDE in all points $[28,29]$. Of course, at the singularities the hypothesis of Cauchy-Kovalevsky theorem are not satisfied.

The aim of this work is to do a complete group classification of the beam equation (1.1), and to report the reduction obtained from the optimal system of subalgebras. In order to find further reductions we also apply the nonclassical method to (1.1). For $f(u)$ linear we find new nonclassical generators unobtainable by Lie classical method. From these reductions we also derive exact solutions. Some of these solutions are compactons solutions, kinks, solutions expressed in terms of Jacobi elliptic functions and Wadati solitons.

\section{Lie Symmetries Classification and Reductions}

The classical method, due originally to Lie (1895) [23], was popularized by Ovsiannikov [27] and presented in a modern form using the jet space theory by Olver [26]. This method 
leads us to those one-parameter group of transformations called classical symmetries that leave the equation unchanged, and hence, they map the set of all solutions to itself. These symmetries are used to reduce the order of ordinary differential equations (ODE's), or to reduce the number of independent variables of PDE's.

In order to apply the classical method to Eq. (1.1) we consider the one-parameter Lie group of infinitesimal transformations in $(x, t, u)$ given by

$$
\begin{aligned}
x^{*} & =x+\epsilon \xi(x, t, u)+\mathcal{O}\left(\epsilon^{2}\right), \\
t^{*} & =t+\epsilon \tau(x, t, u)+\mathcal{O}\left(\epsilon^{2}\right), \\
u^{*} & =u+\epsilon \eta(x, t, u)+\mathcal{O}\left(\epsilon^{2}\right),
\end{aligned}
$$

where $\epsilon$ is the group parameter. One requires that these transformations leave invariant the set of solutions of Eq. (1.1). The associated Lie algebra of infinitesimal symmetries is given by the infinitesimal generator

$$
\mathbf{v}=\xi(x, t, u) \partial_{x}+\tau(x, t, u) \partial_{t}+\eta(x, t, u) \partial_{u}
$$

Since a solution can be used to generate new solutions using different groups, it would be convenient to identify the minimum collection of subgroups that will generate all possible group invariant solutions. Such a collection is called an optimal system and it is constructed by examining the ways in which group invariant solutions transform among themselves through the adjoint operation, [26].

The symmetry variables are found by solving the invariant surface condition

$$
\Phi \equiv \xi(x, t, u) u_{x}+\tau(x, t, u) u_{t}-\eta(x, t, u)=0 .
$$

For PDE's with two independent variables, as it is the beam equation, a single group reduction transforms the PDE into ODE's which are generally easier to solve than the original equation.

We require that the transformations (2.1) leave invariant the set of solutions of equation (1.1). This yields to an overdetermined, linear system of equations for the infinitesimals $\xi(x, t, u), \tau(x, t, u)$ and $\eta(x, t, u)$ and it is accomplished by requiring that the

$$
\left.\operatorname{pr}^{(4)} \mathbf{v}(\Delta)\right|_{\Delta=0}=0
$$

where $\operatorname{pr}^{(4)} \mathbf{v}$ is the 4-th order prolongation of the vector field $\mathbf{v}$.

Applying the classical method to Eq. (1.1) yields to the following system of equations for the infinitesimals

$$
\begin{aligned}
\tau_{t}-2 \xi_{x} & =0, \\
4 \eta_{u x x x}-\xi_{x x x x} & =0, \\
2 \eta_{u x}-3 \xi_{x x} & =0, \\
3 \eta_{u x x}-2 \xi_{x x x} & =0, \\
2 \eta_{t u}-\tau_{t t} & =0, \\
\eta_{t t}+\eta_{x x x x}-f \eta_{u}+f^{\prime} \eta+2 \tau_{t} f & =0,
\end{aligned}
$$


where $\xi=\xi(x)$ and $\tau=\tau(t)$. These equations were generated using the maxima package symmgrp2009.max [8]. By solving system (2.4) we obtain

$$
\xi=k_{1} x+k_{2}, \quad \tau=2 k_{1} t+k_{3}, \quad \eta=k_{4} u+\phi(x, t),
$$

where $k_{i}, i=1, \ldots, 4$, are arbitrary constants, and $f$ and $\phi$ are related by the following condition:

$$
k_{4} u f_{u}+\phi f_{u}+\left(4 k_{1}-k_{4}\right) f+\phi_{x x x x}+\phi_{t t}=0 .
$$

For $f(u)$ arbitrary we get that the associated Lie algebra of infinitesimal symmetries admitted by (1.1) is given by the infinitesimal generators

$$
\mathbf{v}_{1}=\partial_{x}, \quad \mathbf{v}_{2}=\partial_{t}
$$

- For the generator $\lambda \mathbf{v}_{1}+\mathbf{v}_{2}$ we obtain travelling wave reductions

$$
z=x-\lambda t, \quad u(x, t)=h(z)
$$

where $h(z)$ satisfies

$$
h^{\prime \prime \prime \prime}+\lambda^{2} h^{\prime \prime}+f(h)=0 .
$$

From (2.5), according to the form of $f$, we can distinguish different cases for which Eq. (1.1) have extra symmetries. Since Eq. (1.1) has additional symmetries and the reductions that correspond to $\mathbf{v}_{1}$ and $\mathbf{v}_{2}$ have already been derived, we also determine the similarity variables and similarity solutions corresponding to the other generators.

Case 1: If $f(u)=(a u+b)^{n}$, with $a, b \neq 0$ and $n \neq 1$, besides $\mathbf{v}_{1}$ and $\mathbf{v}_{2}$, we obtain the infinitesimal generator

$$
\mathbf{v}_{3}^{1}=x \partial_{x}+2 t \partial_{t}+\frac{4(a u+b)}{a(1-n)} \partial_{u}
$$

The generators of the nontrivial one-dimensional optimal system are the set

$$
\mathbf{v}_{1}, \lambda \mathbf{v}_{1}+\mathbf{v}_{2}, \mathbf{v}_{3}^{1},
$$

where $\lambda \in R$ is an arbitrary constant.

For $\mathbf{v}_{3}^{1}$ we obtain the symmetry reduction

$$
z=x t^{-\frac{1}{2}}, \quad u=t^{\frac{2}{1-n}} h(z)-\frac{b}{a}
$$

where $h(z)$ satisfies

$$
h^{\prime \prime \prime \prime}+\frac{1}{4} z^{2} h^{\prime \prime}+\frac{3 n+5}{4(n-1)} z h^{\prime}+a^{n} h^{n}+\frac{2(n+1)}{(n-1)^{2}} h=0 .
$$


Equation (2.7) does not admit any classical Lie symmetry. However, from particular solutions of Eq. (2.7), we can obtain some exact solutions of (1.1):

- For $a=\sqrt[n]{\frac{-8(n+1)(n+3)(3 n+1)}{c_{1}^{n-1}(n-1)^{4}}}$,

$$
h(z)=c_{1} z^{\frac{4}{1-n}}
$$

is a solution of Eq. (2.7) which leads to the solution of Eq. (1.1)

$$
u(x, t)=c_{1} x^{\frac{4}{1-n}}-\frac{b}{a} .
$$

- For $a=\frac{1}{c_{1}} \sqrt[n]{\frac{-2 c_{1}(n+1)}{(n-1)^{2}}}$ and $h=c_{1}$ we obtain

$$
u(x, t)=c_{1} t^{\frac{2}{1-n}}-\frac{b}{a} .
$$

Case 2: If $f(u)=b \exp (a u)$, with $a, b \neq 0$, besides $\mathbf{v}_{1}$ and $\mathbf{v}_{2}$, the equation has an extra symmetry, namely,

$$
\mathbf{v}_{3}^{2}=x \partial_{x}+2 t \partial_{t}-\frac{4}{a} \partial_{u} .
$$

The generators of the nontrivial one-dimensional optimal system are the set

$$
\mathbf{v}_{1}, \lambda \mathbf{v}_{1}+\mathbf{v}_{2}, \mathbf{v}_{3}^{2},
$$

where $\lambda \in R$ is an arbitrary constant.

For $\mathbf{v}_{3}^{2}$ the similarity variables and similarity solutions are:

$$
z=x t^{-\frac{1}{2}}, \quad u(x, t)=-\frac{2}{a} \ln (t)+h(z),
$$

where $h(z)$ satisfies

$$
h^{\prime \prime \prime \prime}+\frac{z^{2}}{4} h^{\prime \prime}+\frac{3}{4} z h^{\prime}+b \exp (a h(z))+\frac{2}{a}=0 .
$$

Equation (2.9) does not admit any classical Lie symmetry. However, by using particular solutions of the Eq. (2.9), we can obtain some solutions of Eq. (1.1):

- For $b=-\frac{24}{a c_{1}^{a}}$,

$$
h=\ln \left(c_{1} z^{-\frac{4}{a}}\right)
$$

is solution of Eq. (2.9) which leads to the solution of Eq. (1.1)

$$
u(x, t)=\ln \left(c_{1} x^{-\frac{4}{a}}\right) .
$$

- Making the variable change $u=\ln \left(x^{-a} h(z)\right)$ we can find the solution of Eq. (1.1)

$$
u(x, t)=\frac{1}{a} \ln \left(4 a c_{1} \cosh ^{-2}\left[a \sqrt{2 b c_{1}}\left(t+c_{2}\right)\right]\right) .
$$


For completeness, we have included the case in which $f(u)$ is linear.

Case 3: If $f(u)=u, \phi(x, t)$ is any solution to (1.1) and we only consider the subgroup for which $\phi(x, t)=0,[4]$, then, besides $\mathbf{v}_{1}$ and $\mathbf{v}_{2}$, we obtain the infinitesimal generator

$$
\mathbf{v}_{3}^{3}=u \partial_{u}
$$

The generators of the nontrivial one-dimensional optimal system are the set

$$
\mathbf{v}_{1}, \lambda \mathbf{v}_{1}+\mathbf{v}_{2}, \lambda \mathbf{v}_{1}+\alpha \mathbf{v}_{2}+\mathbf{v}_{3}^{3},
$$

where $\lambda, \alpha \in R$ are arbitrary constants.

For $\lambda \mathbf{v}_{1}+\alpha \mathbf{v}_{2}+\mathbf{v}_{3}^{3}$ the similarity variables and similarity solutions are:

$$
z=\alpha x-\lambda t, \quad u(x, t)=h(z) e^{\frac{1}{\alpha} t},
$$

where $h(z)$ satisfies

$$
\alpha^{6} h^{\prime \prime \prime \prime}+\lambda^{2} \alpha^{2} h^{\prime \prime}-2 \lambda \alpha h^{\prime}+\left(1+\alpha^{2}\right) h=0 .
$$

The solution of Eq. (2.14) is

$$
h(z)=\sum_{i=0}^{4} C_{i} e^{w_{i} z},
$$

where $w_{i}$, with $i=1, \ldots, 4$ are the roots of equation

$$
\alpha^{6} w^{4}+\lambda^{2} \alpha^{2} w^{2}-2 \lambda \alpha w+\alpha^{2}+1=0 .
$$

Case 4: If $f(u)=0, \phi(x, t)$ is any solution to (1.1) and we only consider the subgroup for which $\phi(x, t)=0$, then, besides $\mathbf{v}_{1}, \mathbf{v}_{2}$ and $\mathbf{v}_{3}^{3}$, we obtain the infinitesimal generator

$$
\mathbf{v}_{4}=x \partial_{x}+2 t \partial_{t}
$$

The generators of the nontrivial one-dimensional optimal system are the set

$$
\mathbf{v}_{1}, \lambda \mathbf{v}_{1}+\mathbf{v}_{2}, \lambda \mathbf{v}_{3}^{3}+\mathbf{v}_{4},
$$

where $\lambda \in R$ is an arbitrary constant.

For $\lambda \mathbf{v}_{3}^{3}+\mathbf{v}_{4}$ the similarity variables and similarity solutions are:

$$
z=x t^{-\frac{1}{2}}, \quad u(x, t)=t^{\frac{\lambda}{2}} h(z)
$$

where $h(z)$ satisfies

$$
h^{\prime \prime \prime \prime}+\frac{z^{2}}{4} h^{\prime \prime}-\frac{(2 \lambda-3)}{4} z h^{\prime}+\frac{\lambda(\lambda-2)}{4} h=0 .
$$

The general solution of Eq. (2.13) is

$$
\begin{aligned}
h(z)= & C_{1} \mathrm{~F}\left(\frac{2-\lambda}{4},-\frac{\lambda}{4} ; \frac{1}{4}, \frac{1}{2}, \frac{3}{4} ;-\frac{z^{4}}{64}\right)+C_{2} z^{2} \mathrm{~F}\left(\frac{2-\lambda}{4}, \frac{4-\lambda}{4} ; \frac{3}{4}, \frac{5}{4}, \frac{3}{2} ;-\frac{z^{4}}{64}\right) \\
& +C_{3} z^{3} \mathrm{~F}\left(\frac{3-\lambda}{4}, \frac{5-\lambda}{4} ; \frac{5}{4}, \frac{3}{2}, \frac{7}{4} ;-\frac{z^{4}}{64}\right)+C_{4} z \mathrm{~F}\left(\frac{1-\lambda}{4}, \frac{3-\lambda}{4} ; \frac{1}{2}, \frac{3}{4}, \frac{5}{4} ;-\frac{z^{4}}{64}\right),
\end{aligned}
$$

where $\mathrm{F}\left(a_{1}, a_{2} ; b_{1}, b_{2}, b_{3} ; w\right)$ is the generalized hypergeometric function. 
Case 5: If $f(u)=1$, we obtain the infinitesimal generators $\mathbf{v}_{1}, \mathbf{v}_{2}, 4 \mathbf{v}_{3}^{3}+\mathbf{v}_{4}$. The generators of the nontrivial one-dimensional optimal system are the set

$$
\mathbf{v}_{1}, \quad \lambda \mathbf{v}_{1}+\mathbf{v}_{2}, \quad 4 \mathbf{v}_{3}^{3}+\mathbf{v}_{4},
$$

where $\lambda \in R$ is an arbitrary constant.

From $4 \mathbf{v}_{3}^{3}+\mathbf{v}_{4}$ we obtain the symmetry reduction (2.12) with $\lambda=4$ where $h(z)$ satisfies

$$
h^{\prime \prime \prime \prime}+\frac{z^{2}}{4} h^{\prime \prime}-\frac{5}{4} z h^{\prime}+2 h+1=0 .
$$

The general solution of Eq. (2.14) is

$$
\begin{aligned}
h(z)= & -\frac{1}{2}+c_{1} z^{2}+c_{2}\left(z^{4}-12\right) \\
& +c_{3}\left(e^{-\frac{i z^{2}}{4}}\left(-2 z^{3}+20 i z\right)+\frac{1+i}{\sqrt{2}} \sqrt{\pi}\left(-z^{4}+12 i z^{2}+12\right) \operatorname{Erf}\left(\frac{1+i}{2 \sqrt{2}} z\right)\right) \\
& +c_{4}\left(e^{\frac{i z^{2}}{4}}\left(2 i z^{3}-20 z\right)-\frac{1+i}{\sqrt{2}} \sqrt{\pi}\left(z^{4}+12 i z^{2}-12\right) \operatorname{Erf}\left(\frac{-1+i}{2 \sqrt{2}} z\right)\right),
\end{aligned}
$$

where $\operatorname{Erf}(z)$ is the error function [1].

\section{Nonclassical Method}

In [16] Clarkson and Mansfield proposed an algorithm for calculating the determining equations associated with the nonclassical method: the PDE system is augmented with the invariant surface conditions, the nonclassical symmetries are found by seeking the classical symmetries of the augmented system while demanding that the symmetries operator is related to the invariant surface condition. Bilă and Niesen in [3] dropped this requirement. Their procedure consists in reducing the augmented PDE system to its involutive form and then applying the classical Lie method to the reduced PDE, but with an arbitrary symmetry operator which is not related anymore to the invariant surface condition. In [7] Bruzón and Gandarias have extended the algorithm described for Bilă and Niesen to determine the nonclassical symmetries of a PDE for the case $\xi_{p}=0$. The authors observed that for any equation which can be expressed in the form $u_{t}=\mathcal{A}(x, t, u), u_{t t}=\mathcal{A}(x, t, u)$ or $\mathcal{A}(x, y, t, u) u_{y}+u_{t y}+\mathcal{B}(x, y, t, u)=0$, the nonclassical determining equations can be derived by substituting the corresponding functions $\mathcal{A}$ and $\mathcal{B}$, which are given in [7].

To apply the nonclassical method to the beam Eq. (1.1) we require both the equation and the surface condition (2.3) to be invariant under the infinitesimal generator (2.2). We can distinguish two cases:

Case (i): $\tau \neq 0$. We apply the algorithm described in [3]. If $\tau \neq 0$, without loss of generality, we may set $\tau=1$. Substituting

$$
u_{t t}=u_{x} \xi \xi_{x}+2\left(u_{x}\right)^{2} \xi \xi_{u}-\eta u_{x} \xi_{u}-u_{x} \xi_{t}+u_{x x} \xi^{2}-2 \eta_{u} u_{x} \xi-\eta_{x} \xi+\eta \eta_{u}+\eta_{t},
$$

which is obtained from the invariant surface condition $u_{t}=\eta-\xi u_{x}$ in (1.1), we express (1.1) in the equivalent form

$$
u_{x x x x}+\mathcal{A}(x, t, u) u_{x}+\mathcal{B}(x, t, u) u_{x x}+\mathcal{C}(x, t, u)\left(u_{x}\right)^{2}+\mathcal{D}(x, t, u)=0,
$$


where $\mathcal{A}=\left(-2 \eta \xi_{u}-\eta_{u} \xi+\eta \eta_{x}-\eta_{t}\right), \mathcal{B}=\eta^{2}, \mathcal{C}=2 \eta \eta_{u}$ and $\mathcal{D}=-\eta \xi_{x}+\xi \xi_{u}+\xi_{t}+f(u)$. We apply the classical Lie method to (3.1). The infinitesimal generator is

$$
V=\xi_{1}(x, t, u) \partial_{x}+\xi_{2}(x, t, u) \partial_{t}+\phi(x, t, u) \partial_{u} .
$$

Substituting $\xi_{1}=\xi, \xi_{2}=1, \phi=\eta$ and $\mathcal{A}, \mathcal{B}, \mathcal{C}$ and $\mathcal{D}$ we obtain the determining equations of the nonclassical method

$$
\begin{aligned}
\xi_{u} & =0, \\
\eta_{u u} & =0, \\
2 \eta_{u x}-3 \xi_{x x} & =0, \\
3 \eta_{u x x}+\xi \xi_{t}-2 \xi_{x x x}+2 \xi^{2} \xi_{x} & =0, \\
2 \xi \eta_{u t}-4 \eta_{u x x x}+2 \xi_{t} \eta_{u}+8 \xi \xi_{x} \eta_{u}+\xi_{t t}+2 \xi_{x} \xi_{t}+\xi_{x x x x}-4 \xi\left(\xi_{x}\right)^{2} & =0, \\
\eta_{t t}+4 \xi_{x} \eta_{t}+\eta_{x x x x}-2 \xi_{t} \eta_{x}-4 \xi \xi_{x} \eta_{x}+2 \eta \eta_{u t}+4 \xi_{x} \eta \eta_{u}-f \eta_{u}+f_{u} \eta+4 \xi_{x} f & =0 .
\end{aligned}
$$

In this case we do not obtain new symmetries.

Case (ii): $\tau=0$. For this case we proceed in the form described in [7]. We consider, without loss of generality, $\xi=1$. By using the invariant surface condition $u_{x}=\eta$ and its derivatives, the equivalent form of (1.1) is

$$
u_{t t}=\mathcal{A},
$$

where $\mathcal{A}=-f-\eta_{x x x}-\eta_{u} \eta_{x x}-3 \eta \eta_{u u} \eta_{x}-3 \eta_{u x} \eta_{x}-\left(\eta_{u}\right)^{2} \eta_{x}-\eta^{3} \eta_{u u u}-3 \eta^{2} \eta_{u u x}-4 \eta^{2} \eta_{u} \eta_{u u}-$ $3 \eta \eta_{u x x}-5 \eta \eta_{u} \eta_{u x}-\eta\left(\eta_{u}\right)^{3}$.

Substituting $\mathcal{A}$ into the determining equations of the nonclassical method, which were obtained in [7]

$$
\begin{aligned}
\eta_{u u} & =0, \\
\eta_{t u} & =0, \\
\mathcal{A} \eta_{u}+\eta_{t t}-\mathcal{A}_{u} \eta-\mathcal{A}_{x} & =0 .
\end{aligned}
$$

we obtain the determining equations for Eq. (1.1) for the infinitesimal $\eta$

$$
\begin{aligned}
\eta_{u u} & =0, \\
\eta_{t u} & =0, \\
\eta_{x x x x}+4 \eta_{u x} \eta_{x x}+6 \eta_{u x x} \eta_{x}+4 \eta_{u} \eta_{u x} \eta_{x}+4 \eta \eta_{u x x x}+6 \eta \eta_{u} \eta_{u x x} & \\
+8 \eta\left(\eta_{u x}\right)^{2}+4 \eta\left(\eta_{u}\right)^{2} \eta_{u x}-f \eta_{u}+\eta_{t t}+f_{u} \eta & =0 .
\end{aligned}
$$

From (3.4) and (3.5) we deduce

$$
\eta=\alpha(x) u+\beta(x, t)
$$

If $\alpha=0$ and $\beta=0$ the symmetries obtained are classical symmetries.

If $\alpha \neq 0$ or $\beta \neq 0$, substituting (3.7) in (3.6) we obtain that $f=a u+b$ with $a \neq 0$. In this case, $\alpha$ and $\beta$ must satisfy the following equations:

$$
\begin{aligned}
\alpha^{\prime \prime \prime \prime}+4 \alpha \alpha^{\prime \prime \prime}+10 \alpha^{\prime} \alpha^{\prime \prime}+6 \alpha^{2} \alpha^{\prime \prime}+12 \alpha\left(\alpha^{\prime}\right)^{2}+4 \alpha^{3} \alpha^{\prime}=0 \\
\beta_{x x x x}+4 \alpha^{\prime} \beta_{x x}+6 \alpha^{\prime \prime} \beta_{x}+4 \alpha \alpha^{\prime} \beta_{x}+\beta_{t t}+a \beta+4 \alpha^{\prime \prime \prime} \beta+6 \alpha \alpha^{\prime \prime} \beta \\
+8\left(\alpha^{\prime}\right)^{2} \beta+4 \alpha^{2} \alpha^{\prime} \beta-\alpha b=0 .
\end{aligned}
$$


Solving the first equation of (3.8), we obtain that if $\alpha=\frac{k}{x+x_{0}}$, with $k=1,2$ or 3 , the system (3.8) is reduced to equation

$$
\begin{array}{r}
{\left[-4 k(k-3)(k-2)+a\left(x+x_{0}\right)^{4}\right] \beta+\left(x+x_{0}\right)\left[-b k\left(x+x_{0}\right)^{2}\right.} \\
\left.-4 k(k-3) \beta_{x}+\left(x+x_{0}\right)\left[-4 k \beta_{x x}+\left(x+x_{0}\right)^{2}\left(\beta_{t t}+\beta_{x x x x}\right)\right]\right]=0 .
\end{array}
$$

- For $k=1, x_{0}=0$, Eq. (3.9) can be reduced to the equation $x^{4}\left(\beta_{x x x x}+\beta_{t t}+a \beta\right)-b x^{3}-$ $4 x^{2} \beta_{x x}+8 x \beta_{x}-8 \beta=0$. We find the solutions:

- If $\beta=0$ and $b=0$ the symmetry $\xi=1, \tau=0$, and $\eta=\frac{u}{x}$ does not verify the determining equations obtained by the classical Lie method. Therefore we obtain the nonclassical symmetry reduction

$$
w=t, \quad u=x h(t)
$$

where $h(t)$ satisfies the ODE

$$
h^{\prime \prime}+a h=0 .
$$

Hence we obtain for the beam equation (1.1), with $f(u)=a u+b$ and $a \neq 0$, the following exact solutions:

$$
\begin{aligned}
& u(x, t)=c_{1} x \sin (\sqrt{a} t)+c_{2} x \cos (\sqrt{a} t), \\
& u(x, t)=c_{1} x \cosh (\sqrt{-a} t)+c_{2} x \sinh (\sqrt{-a} t) .
\end{aligned}
$$

- If $\beta=\frac{b}{a x}+\frac{k_{1}}{x} \exp (\sqrt[4]{-a} x)(-1+\sqrt[4]{-a} x)+\frac{k_{2}}{x} \exp (-\sqrt[4]{-a} x)(1+\sqrt[4]{-a} x)$.

The symmetry $\xi=1, \tau=0$ and

$$
\eta=\frac{u}{x}+\frac{b}{a x}+\frac{k_{1}}{x} \exp (\sqrt[4]{-a} x)(-1+\sqrt[4]{-a} x)+\frac{k_{2}}{x} \exp (-\sqrt[4]{-a} x)(1+\sqrt[4]{-a} x),
$$

does not verify the determining equation obtained by the classical Lie method. If we take $\beta=\frac{b}{a x}+\frac{k_{1}}{x} \exp (\sqrt[4]{-a} x)(-1+\sqrt[4]{-a} x)$, the transformation

$$
w=t, \quad u=x h(t)-\frac{b}{a}+k_{1} \exp (\sqrt[4]{-a} x)
$$

reduces Eq. (1.1) to Eq. (3.11), and we obtain the following exact solution for the beam Eq. (1.1):

$$
u(x, t)=x^{2}\left(c_{1} \cosh (\sqrt{-a} t)+c_{2} \sinh (\sqrt{-a} t)\right)-\frac{b}{a}+k_{1} \exp (\sqrt[4]{-a} x)
$$

- For $k=2, x_{0}=0$, Eq. (3.9) can be written as

$$
x^{3}\left(a \beta+\beta_{t t}+\beta_{x x x x}\right)+8\left(\beta_{x}-x \beta_{x x}\right)-2 b x^{2}=0 .
$$

For $b=0$ a solution is

$$
\beta=\left(k_{1} \cos (\sqrt{a} t)+k_{2} \sin (\sqrt{a} t)\right)\left(k_{3}+k_{4} x^{2}+k_{5} x^{5}+k_{6} x^{-1}\right) .
$$


The symmetry $\xi=1, \tau=0$ and

$$
\eta=\frac{2 u}{x}+\left(k_{1} \cos (\sqrt{a} t)+k_{2} \sin (\sqrt{a} t)\right)\left(k_{3}+k_{4} x^{2}+k_{5} x^{5}+k_{6} x^{-1}\right),
$$

does not verify the determining equation obtained by the classical Lie method. The transformation

$$
y=t, u=x^{2} h(t)-\frac{1}{4}\left(2 k_{6}+4 k_{3} x-4 k_{4} x^{3}-k_{5} x^{6}\right)\left(k_{1} \cos (\sqrt{a} t)+k_{2} \sin (\sqrt{a} t)\right),
$$

with $k_{1}, \ldots, k_{6}$ arbitrary constants, reduces Eq. (1.1) to equation

$$
h^{\prime \prime}+a h+90 k_{5}\left(k_{1} \cos (\sqrt{a} t)+k_{2} \sin (\sqrt{a} t)\right)=0 .
$$

We obtain a new exact solution for the beam Eq. (1.1):

$$
\begin{aligned}
u(x, t)= & x^{2}\left(c_{1} \cos (\sqrt{a} t)+c_{2} \sin (\sqrt{a} t)\right)+\frac{45 \sqrt{a} k_{5} x^{2} t}{a}\left(k_{2} \cos (\sqrt{a} t)-k_{1} \sin (\sqrt{a} t)\right) \\
& -\frac{1}{4}\left(2 k_{6}+4 k_{3} x-4 k_{4} x^{3}-k_{5} x^{6}\right)\left(k_{1} \cos (\sqrt{a} t)+k_{2} \sin (\sqrt{a} t)\right)
\end{aligned}
$$

- For $k=3$ and $x_{0}=0$ Eq. (3.9) can be written as

$$
-3\left(b x+4 \beta_{x x}\right)+x^{2}\left(a \beta+\beta_{t t}+\beta_{x x x x}\right)=0 .
$$

We find

$$
\begin{aligned}
\beta= & \frac{3 c_{2}}{a x}+\left(k_{4} \sin \left(\sqrt{a+k_{1}} t\right)+k_{5} \cos \left(\sqrt{a+k_{1}} t\right)\right) \\
& \left.\times\left(k_{2}\left(\sqrt[4]{k_{1}}+\frac{3}{x}\right) \exp \left(-\sqrt[4]{k_{1}} x\right)+k_{3}\left(\sqrt[4]{k_{1}}-\frac{3}{x}\right) \exp \left(\sqrt[4]{k_{1}} x\right)\right)\right),
\end{aligned}
$$

with $k_{1}, k_{2}, \ldots, k_{5}$ arbitrary constants. The symmetry $\xi=1, \tau=0$ and

$$
\begin{aligned}
\eta= & \frac{3 u}{x}+\frac{3 b}{a x}+\left(k_{4} \sin \left(\sqrt{a+k_{1}} t\right)+k_{5} \cos \left(\sqrt{a+k_{1}} t\right)\right) \\
& \times\left(k_{2}\left(\sqrt[4]{k_{1}}+\frac{3}{x}\right) \exp \left(-\sqrt[4]{k_{1}} x\right)+k_{3}\left(\sqrt[4]{k_{1}}-\frac{3}{x}\right) \exp \left(\sqrt[4]{k_{1}} x\right)\right),
\end{aligned}
$$

does not verify the determining equation obtained by the classical Lie method.

If we take

$$
\beta=\frac{3 b}{a x}+k_{2} \sin \left(\sqrt{a+k_{1}} t\right)\left(\sqrt[4]{k_{1}}+\frac{3}{x}\right) \exp \left(-\sqrt[4]{k_{1}} x\right)
$$

the transformation $y=t, u=x^{3} h(t)-\frac{b}{a}+k_{2} \sin \left(\sqrt{a+k_{1}} t\right) \exp \left(-\sqrt[4]{k_{1}} x\right)$ reduces Eq. (1.1) to Eq. (3.11).

The solution for the beam Eq. (1.1) is:

$$
u(x, t)=x^{3}\left(c_{1} \cos (\sqrt{a} t)+c_{2} \sin (\sqrt{a} t)\right)-\frac{b}{a}+k_{2} \sin \left(\sqrt{a+k_{1}} t\right) \exp \left(-\sqrt[4]{k_{1}} x\right)
$$




\section{Travelling Waves Solutions}

Due to the fact that the Jacobian elliptic functions are solution of the equation of Jacobi

$$
\left(H^{\prime}\right)^{2}=r+p H^{2}+q H^{4}
$$

with $r, p$ and $q$ constants, we can look for travelling wave solutions of the generalized beam equation when $f(u)$ is a finite linear sum of power functions.

Then the reduced ODE (2.6) becomes

$$
h^{\prime \prime \prime \prime}+\lambda^{2} h^{\prime \prime}+\sum_{i=1}^{n} \alpha_{i} h^{\beta_{i}}=0 .
$$

By using derivation and taking into account that $\mathrm{cn}^{2}(z, m)=1-\operatorname{sn}^{2}(z, m)=1-\left(\frac{h}{\alpha}\right)^{\frac{2}{\beta}}$ and $\mathrm{dn}^{2}(z, m)=1-m \operatorname{sn}^{2}(z, m)=1-m\left(\frac{h}{\alpha}\right)^{\frac{2}{\beta}}$, see [1], we obtain Case (i): For

$$
\begin{gathered}
h(z)=\alpha \operatorname{sn}^{\beta}(z, m) . \\
f(h)=\alpha_{1} h^{\frac{4}{\beta}+1}+\alpha_{2} h^{\frac{2}{\beta}+1}+\alpha_{3} h^{-\frac{4}{\beta}+1}+\alpha_{4} h^{-\frac{2}{\beta}+1}+\alpha_{5} h,
\end{gathered}
$$

where

$$
\begin{aligned}
& \alpha_{1}=-\frac{\beta(\beta+1)(\beta+2)(\beta+3) m^{2}}{\alpha^{\frac{4}{\beta}}}, \\
& \alpha_{2}=-\frac{\beta(\beta+1) m\left(\lambda^{2}-2 \beta^{2} m-4 \beta m-4 m-2 \beta^{2}-4 \beta-4\right)}{\alpha^{\frac{2}{\beta}}}, \\
& \alpha_{3}=-\alpha^{\frac{4}{\beta}}(\beta-3)(\beta-2)(\beta-1) \beta, \\
& \alpha_{4}=-\alpha^{\frac{2}{\beta}}(\beta-1) \beta\left(\lambda^{2}-2 \beta^{2} m+4 \beta m-4 m-2 \beta^{2}+4 \beta-4\right), \\
& \alpha_{5}=\beta^{2}\left(m \lambda^{2}+\lambda^{2}-\beta^{2} m^{2}-4 \beta^{2} m-10 m-\beta^{2}\right) .
\end{aligned}
$$

Hence, an exact solution of Eq. (1.1), where $f(u)$ is obtained substituting $h$ by $u$ in (4.4), is

$$
u(x, t)=\alpha \operatorname{sn}^{\beta}(x-\lambda t, m) .
$$

Case (ii): For

$$
h(z)=\alpha \operatorname{dn}^{\beta}(z, m)
$$

in a similar way we can write

$$
f(h)=\gamma_{1} h^{-\frac{2}{\beta}+1}+\gamma_{2} h+\gamma_{3} h^{\frac{2}{\beta}+1}+\gamma_{4} h^{-\frac{4}{\beta}+1}+\gamma_{5} h^{\frac{4}{\beta}+1},
$$


where

$$
\begin{aligned}
& \gamma_{1}=-\alpha^{\frac{2}{\beta}}(\beta-1) \beta(m-1)\left(\lambda^{2}-2 \beta^{2} m+4 \beta m-4 m+4 \beta^{2}-8 \beta+8\right), \\
& \gamma_{2}=\beta^{2}\left(m \lambda^{2}-2 \lambda^{2}-\beta^{2} m^{2}+6 \beta^{2} m+10 m-6 \beta^{2}-10\right), \\
& \gamma_{3}=\frac{\beta(\beta+1)\left(\lambda^{2}-2 \beta^{2} m-4 \beta m-4 m+4 \beta^{2}+8 \beta+8\right)}{\alpha^{\frac{2}{\beta}}} \\
& \gamma_{4}=-\alpha^{\frac{4}{\beta}}(\beta-3)(\beta-2)(\beta-1) \beta(m-1)^{2} \\
& \gamma_{5}=-\frac{\beta(\beta+1)(\beta+2)(\beta+3)}{\alpha^{\frac{4}{\beta}}} .
\end{aligned}
$$

Hence, an exact solution of Eq. (1.1), where $f(u)$ is obtained substituting $h$ by $u$ in $(4.9)$, is

$$
u(x, t)=\alpha \operatorname{dn}^{\beta}(x-\lambda t, m) .
$$

In the same way, we can obtain functions $f(h)$ for which the functions $\alpha \mathrm{cn}^{\beta}(z, m)$, and other Jacobian elliptic functions are solutions of Eq. (2.6), as well as $\alpha z^{\beta}$ and $\alpha \exp (z)$.

In the following we give some solutions with physical interest:

- Setting $\alpha=\beta=1, \lambda=-2$ and $m=0.9999$ in (4.4)-(4.6), we get

$$
f(h)=-23.9952 h^{5}+31.9948 h^{3}-7.99879 h,
$$

where $h=\operatorname{sn}(z, 0.9999)$ is a solution of $(2.6)$. Hence, we obtain that

$$
u(x, t)=\operatorname{sn}(x+2 t, 0.9999),
$$

is a solution of Eq. (1.1) with $f(u)=-23.9952 u^{5}+31.9948 u^{3}-7.99879 u$. In Fig. 1 we plot solution (4.14) which is an almost square wave.

- Setting $m=1, \lambda=-\frac{1}{2}, \alpha=\frac{1}{4}$ and $\beta=1$ in (4.4)-(4.6) we get

$$
f(h)=-6144 h^{5}+632 h^{3}-\frac{31}{2} h
$$

where $h(z)=\frac{1}{4} \operatorname{sn}(z, 1)$ is a solution of (2.6). Taking into account that $\operatorname{sn}(z, 1)=\tanh (z)$,

$$
u(x, t)=\frac{1}{4} \tanh \left(x+\frac{1}{2} t\right),
$$

is a solution of (1.1) with $f(u)=-6144 u^{5}+632 u^{3}-\frac{31}{2} u$. In Fig. 2 we plot solution (4.15) which is a kink solution.

- Compactons were introduced by Rosenau and Hyman [29] as a class of solitary wave solutions with compact support. In order to obtain a compacton solution $f(y)$ for a differential equation, $f$ must be a non identically null solution in a compact set $Q$, and $f$ must be null when $y \notin Q$.

For instance, we can find a 2-hump compacton in the following way.

Let us observe that in (2.6), we can obtain the solution $h=0$ if $f(0)=0$.

If $m \in \mathbb{N}, h=A \sin ^{m}(\gamma z)$ for $|z| \leq \frac{\pi}{\gamma}$, and $h=0$ elsewhere, then $h \notin \mathcal{C}^{\infty}(\mathbb{R})$ but $h$ have a jump discontinuity for the $m$-order derivative at the points $z= \pm \frac{\pi}{\gamma}$. 


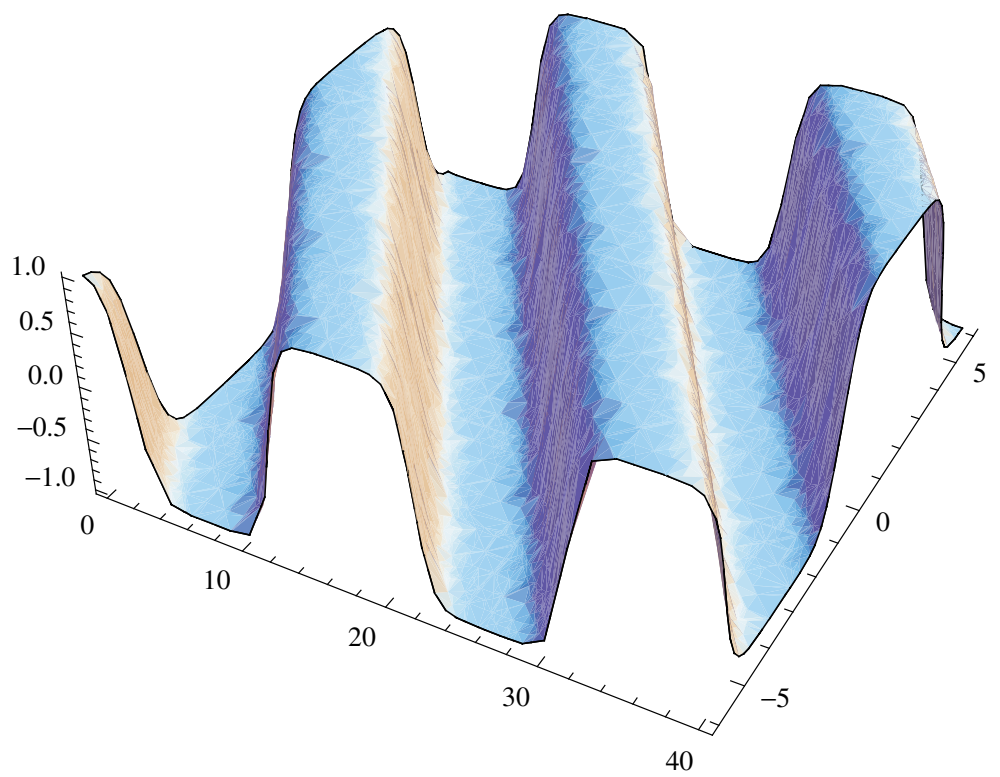

Fig. 1. Solution (4.14).

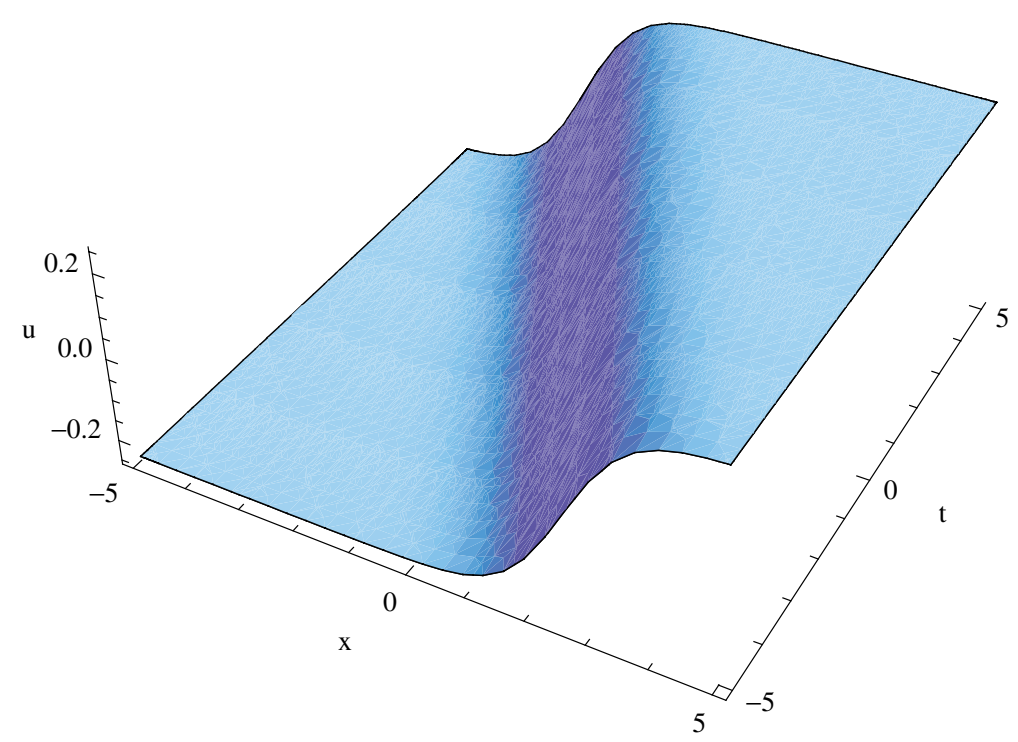

Fig. 2. Solution (4.15).

It can be shown that for $m \geq 6$ the function $h$ is regular enough to be a solution for Eq. (2.6), in the classical sense, by choosing an adequate function $f$. For instance we make $m=6$ and

$$
f(h)=6 \gamma^{2} h^{\frac{1}{3}}\left(60 A^{\frac{2}{3}} \gamma^{2}+6 h^{\frac{2}{3}}\left(36 \gamma^{2}-\lambda^{2}\right)+5 A^{\frac{1}{3}} h^{\frac{1}{3}}\left(\lambda^{2}-52 \gamma^{2}\right)\right) .
$$

Because the Jacobi elliptic function $\operatorname{sn}(z, 0)=\sin (z)$ we can relate this choice of $f$ with the one given in (4.4)-(4.6) with $m=0, \alpha=A$ and $\beta=m$. 
We observe that $h(z)$ is a solution of (2.6) with this choice of $f$ for $|z|<\frac{\pi}{\gamma}$ and for $|z|>\frac{\pi}{\gamma}$. For $z= \pm \frac{\pi}{\gamma}$ the ODE's existence and uniqueness theorem fails: $f(h)$ is a continuous function but it is not a lipschitzian function for $h=0$. Nevertheless the solution $h$ is smooth enough $\left(h \in \mathcal{C}^{5}(R)\right)$ in order to satisfy $(2.6)$. [28,29]. Hence, we obtain that

$$
u(x, t)= \begin{cases}A \sin ^{6}(\gamma(x-\lambda t)), & |x-\lambda t| \leq \frac{\pi}{\gamma}, \\ 0, & |x-\lambda t|>\frac{\pi}{\gamma},\end{cases}
$$

with $f(h)=6 \gamma^{2} h^{\frac{1}{3}}\left(60 A^{\frac{2}{3}} \gamma^{2}+6 h^{\frac{2}{3}}\left(36 \gamma^{2}-\lambda^{2}\right)+5 A^{\frac{1}{3}} h^{\frac{1}{3}}\left(\lambda^{2}-52 \gamma^{2}\right)\right)$, is a compacton solution of (1.1). In Fig. 3 we plot the solution (4.16) with $A=1, \gamma=\frac{1}{4}$ and $\lambda=1$ which is a sine-type double compacton solution.

- Setting $m=\lambda=\alpha=1$ and $\beta=2$ in (4.9)-(4.10) we get

$$
f(h)=-120 h^{3}+126 h^{2}-20 h,
$$

where $h(z)=\operatorname{dn}^{2}(z, 1)$ is a solution of (2.6). Taking into account that $\operatorname{dn}(z, 1)=\operatorname{sech}(z)$,

$$
u(x, t)=\operatorname{sech}^{2}(x-t)
$$

is an exact solution of (1.1) with $f(u)=-120 u^{3}+126 u^{2}-20 u$. In Fig. 4 we plot solution (4.17) which is a soliton solution.

- The Wadati functions are functions of the form $W=\frac{d}{d x}\left(2 \arctan \left(\frac{n_{1} \sin \left(n_{2} x\right)}{n_{2} \cosh \left(n_{1} x\right)}\right)\right)$, where $n_{1}, n_{2}$ are gaussian integers, i.e. complex numbers whose real and imaginary parts are integers. Since these functions can be written in terms of trigonometric or hyperbolic functions, some algebraic relations between the functions and their derivatives could be investigated. So, we will try to find some specific forms of $f$ for which we obtain solutions in Wadati

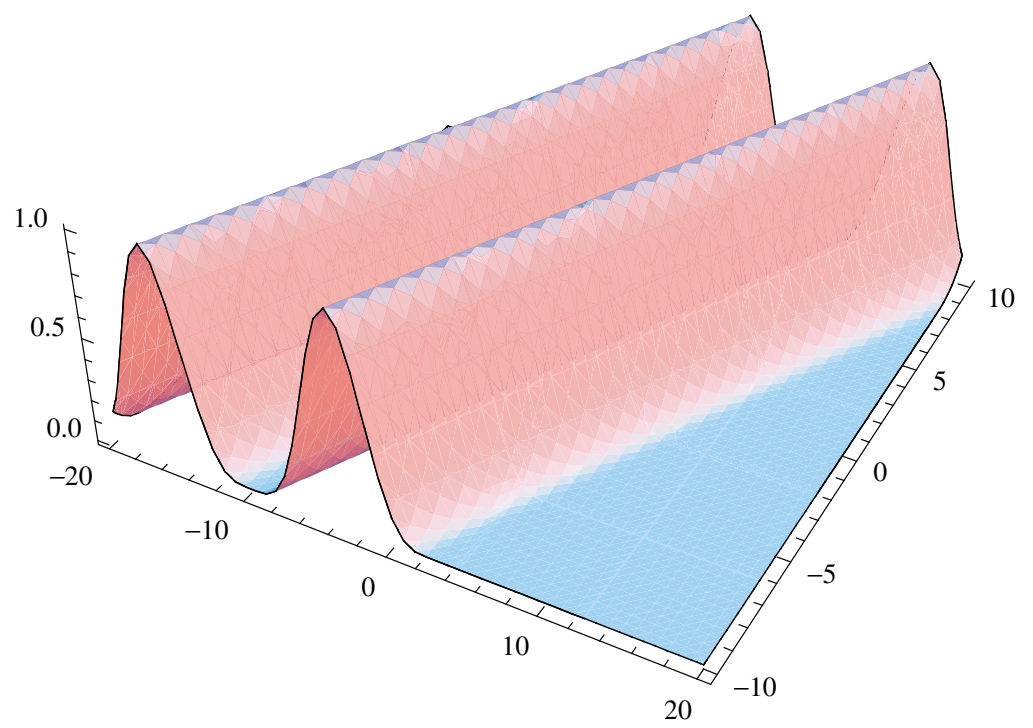

Fig. 3. Solution (4.16) for $\gamma=\frac{1}{4}$. 


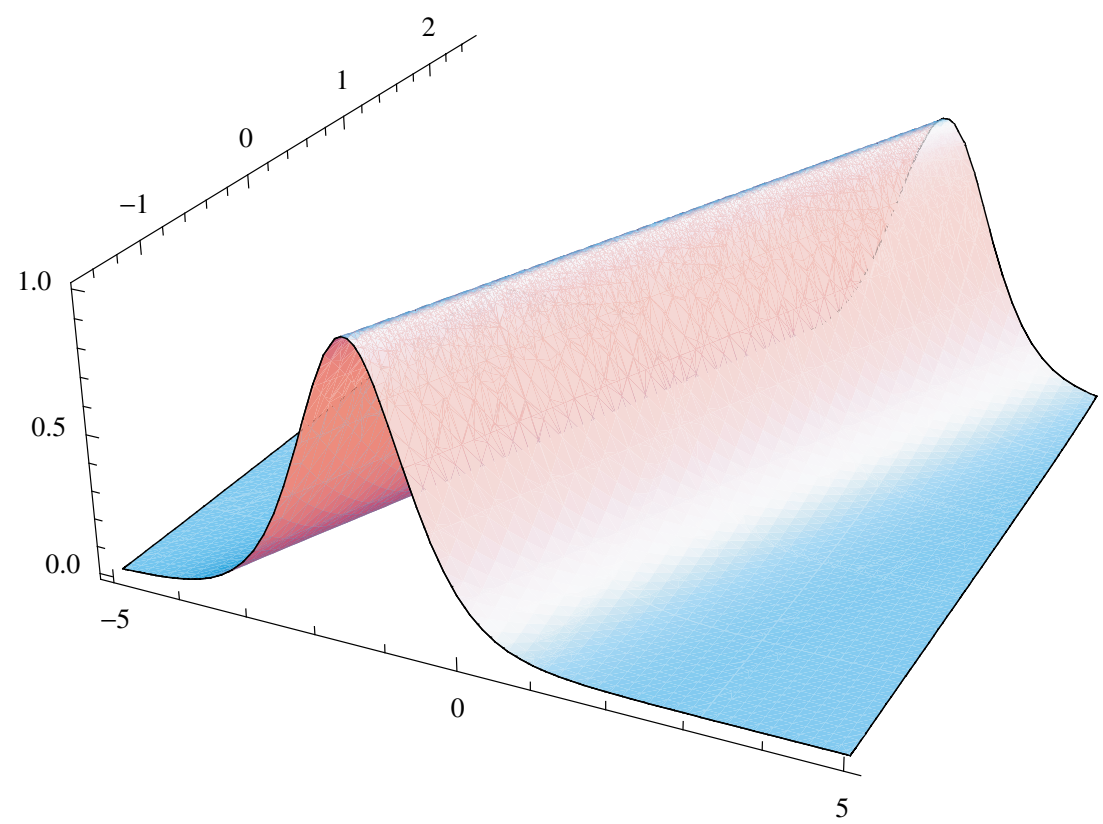

Fig. 4. Solution (4.17).

functions. For $n_{1}=2, n_{2}=i$

$$
h=\frac{4(\cosh (3 z)-3 \cosh (z))}{3-4 \cosh (2 z)-\cosh (4 z)} .
$$

Setting $\cosh (z)=w$ we get

$$
h=\frac{12 w-8 w^{3}}{-3+4 w^{4}}
$$

Substituting (4.19) into (2.6) we obtain

$$
\begin{aligned}
f= & \frac{4 w}{\left(4 w^{4}-3\right)^{5}}\left(512 w^{18}-72448 w^{16}+563712 w^{14}-2240256 w^{12}+4609728 w^{10}-5451552 w^{8}\right. \\
& +4077216 w^{6}-1854576 w^{4}+414882 w^{2}+\left(3-4 w^{4}\right)^{2}\left(2 w ^ { 2 } \left(16 w^{8}-248 w^{6}+552 w^{4}\right.\right. \\
& \left.\left.\left.-684 w^{2}+441\right)-135\right) \lambda^{2}-48843\right)
\end{aligned}
$$

where $w=w(h)$ is given in implicit form in (4.19). Hence, we obtain that

$$
u(x, t)=\frac{4(\cosh (3(x-\lambda t))-3 \cosh (x-\lambda t))}{3-4 \cosh (2(x-\lambda t))-\cosh (4(x-\lambda t))}
$$

is an exact solution for Eq. (1.1). In Fig. 5 we plot solution (4.20) with $\lambda=2$

- When we make $f(h)=\frac{1}{4}\left(a^{2}+\lambda^{2}\right)^{2} h$ the Eq. (2.6) admits the solutions of the damped vibration equation $h^{\prime \prime}+a h^{\prime}+b h=0$ with $b=\frac{1}{2}\left(a^{2}+\lambda^{2}\right)$, i.e. $h^{\prime \prime}+a h^{\prime}+\frac{1}{2}\left(a^{2}+\lambda^{2}\right) h=0$. In the general case this corresponds to an undamped angular frequency equal to $\sqrt{\frac{a^{2}+\lambda^{2}}{2}}$ and a damping factor equal to $\frac{a}{\sqrt{2\left(a^{2}+\lambda^{2}\right)}}$ so the solutions are underdamped, corresponding to an 


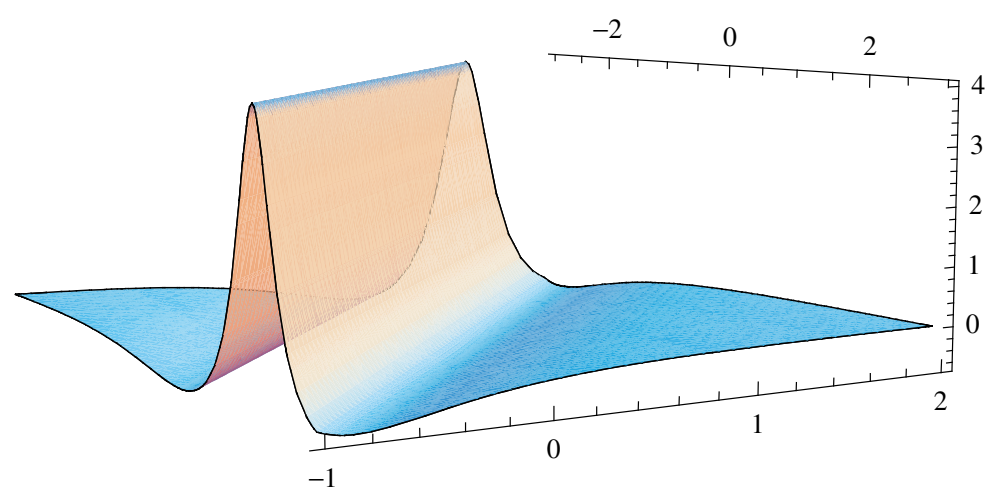

Fig. 5. Solution (4.20) with $\lambda=2$.

oscillatory motion with an exponential decay in the amplitude. When $a=0$ making $f(h)=$ $-b\left(b+\lambda^{2}\right) h$ the Eq. (2.6) admits the solutions of the free vibration equation $h^{\prime \prime}+b h=0$ as $b>0$. When $a=0$ and $\lambda=0$, that correspond to stationary solutions, the equation becomes $h^{\prime \prime}=0$.

\section{Conclusions}

In this paper, the complete Lie group classification for the beam equation (1.1) has been obtained. We have constructed the optimal system and have derived the corresponding reduced equations. We have used an algorithm described by Bilă and Niesen [3] to determine nonclassical symmetries and extended by Bruzón and Gandarias [7]. By using this algorithm, for $f$ linear, we have derived new symmetry generators for equation (1.1). We have derived new travelling wave solutions. Among them we have found solitons, kinks, anti-kinks, two hump compactons and Wadati solitons. The new solutions can display several types of coherent structures.

\section{Acknowledgments}

The support of DGICYT project MTM2009-11875, Junta de Andalucía group FQM-201 and project P06-FQM-01448 are gratefully acknowledged.

\section{References}

[1] M. Abramowitz and I. A. Stegun, Handbook of Mathematical Functions (Dover, New York, 1972).

[2] N. Sukhomlin and J. Alvarez, Simetrías y nuevas soluciones de la ecuación de vibraciones de una viga elástica, Ingeniería y Ciencia 5(9) (2009) 25-43.

[3] N. Bil $A$ and J. Niesen, On a new procedure for finding nonclassical symmetries, J. Symb. Comp. 38 (2004) 1523-1533.

[4] G. W. Bluman and J. D. Cole, The general similarity solution of the heat equation, J. Math. Mech. 18 (1969) 1025-1042.

[5] G. W. Bluman and S. Kumei, Symmetries and Differential Equations (Springer-Verlag, New York, 1989).

[6] M. S. Bruzón, J. C. Camacho and J. Ramírez, Modelo de vibraciones de una viga. Reducciones por simetrías, in Proc. CISCI: Int. Conf. Education 8 Information Systems (Florida, 2004), $168-193$. 
[7] M. S. Bruzón and M. L. Gandarias, Applying a new algorithm to derive nonclassical symmetries, Commun. Nonlinear Sci. Numer. Simul. 13 (2008) 517-523.

[8] B. Champagne, W. Hereman and P. Winternitz, The computer calculation of Lie point symmetries of large systems of differential equations, Comput. Phys. Commun. 66 (1991) 319-340.

[9] Y. S. Choy, K. S. Jen and P. J. McKenna, The structure of the solution set for periodic oscillations in a suspension bridge model, IMA J. Appl. Math. 47 (1991) 283-306.

[10] A. R. Champreys, P. J. McKenna and P. A. Zegeling, Solitary waves in nonlinear beam equations: Stability, fission and fusion, Nonlinear Dynam. 21 (2000) 31-53.

[11] Y. Chen and P. J. McKenna, Traveling waves in a nonlinearly suspended beam: Theoretical results and numerical observations, J. Differential Equations 136 (1997) 325-355.

[12] Y. Chen and P. J. McKenna, Traveling waves in a nonlinearly suspended beam: some computational results and four open questions, Phil. Trans. R. Soc. Lond. A 355 (1997) 2175-2184.

[13] Q. H. Choi and T. Jung, The study of a nonlinear suspension bridge equation by a variational reduction method, Appl. Anal. 50 (1993) 73-92.

[14] S. H. Doole and S. J. Hogan, The nonlinear dynamics of suspension bridges under harmonic forcing, Applied Nonlinear Mathematics Report (1996), 94.4.

[15] S. H. Doole and S. J. Hogan, A piecewise linear suspension bridge model nonlinear dynamics and orbit continuation, Dynam. Stabil. Syst. 11 (1996) 19-47.

[16] P. A. Clarkson and E. L. Mansfield, Algorithms for the nonclassical method of symmetry reductions, SIAM J. Appl. Math. 55 (1994) 1693-1719.

[17] P. A. Clarkson, Nonclassical symmetry reductions of the boussinesq equation, Chaos Solitons, Fractals 5 (1995) 2261-2301.

[18] P. A. Clarkson and T. J. Priestley, Symmetries of a class of nonlinear fourth order partial differential equations, J. Nonlinear Math. Phys. 6 (1999) 66-98.

[19] L. D. Humphreys, Numerical mountain pass solutions of a suspension bridge equation, Nonlinear Anal. TMA 35 (1997) 1811-1826.

[20] L. D. Humphreys and P. J. McKenna, Multiple periodic solutions for a nonlinear suspension bridge equation, IMA J. Appl. Math. 63 (1999) 37-49.

[21] A. C. Lazer and P. J. McKenna, Large Amplitude periodic oscillation in suspension bridges: some new connections with nonlinear analysis, SIAM Rev. 32 (1990) 537-578.

[22] D. Levi and P. Winternitz, Nonclassical symmetry reduction: example of the Boussinesq equation, J. Phys. A 22 (1989) 2915-2924.

[23] S. Lie, Vorlesungen Ber Differentialgleishungen Mit Bekannten Infinitesimalen Transformationes (Teuber, Leipzig, reprinted by Chelsea, New York, 1891).

[24] P. J. McKenna and W. Walter, Nonlinear oscillations in a suspension bridge, Arch. Ration. Mech. Anal. 98 (1987) 167-177.

[25] P. J. McKenna and W. Walter, Travelling waves in a suspension bridges, SIAM J. Appl. Math. 50 (1990) 703-715.

[26] P. J. Olver, Applications of Lie Groups to Differential Equations (Springer-Verlag, New York, 1986).

[27] L. V. Ovsiannikov, Group Analysis of Differential Equations (New York: Academic, 1982).

[28] P. Rosenau, What is ... a compacton? Notices Amer. Math. Soc. 52 (2005) 738-739.

[29] P. Rosenau and J. M. Hyman, Compactons: Solitons with finite wavelengths, Phys. Rev. Lett. 70(5) (1993) 564-571. 